\title{
Conexões de conhecimentos especializados de professores de Biologia sobre temas do
}

\section{Ensino Médio}

\author{
Connections to specialized knowledge of Biology teachers on High School subjects \\ Conexiones del conocimientos especializados de profesores de Biología en temas de la Escuela
}

Secundaria

Recebido: 09/09/2021 | Revisado: 17/09/2021 | Aceito: 19/09/2021 | Publicado: 20/09/2021

\author{
Marcela Marques \\ ORCID: https://orcid.org/0000-0001-5537-0461 \\ Secretaria de Estado de Educação SEDUC, Brasil \\ E-mail: m.marquesbio@gmail.com \\ Susel Taís Coelho Soares \\ ORCID: https://orcid.org/0000-0003-4681-968X \\ Instituto Federal de Mato Grosso, Brasil \\ E-mail: suseltais@gmail.com \\ Joseany Sebastiana Silva Moreira \\ ORCID: https://orcid.org/0000-0002-1473-8386 \\ Secretaria de Estado de Educação SEDUC, Brasil \\ E-mail: joseanymoreira2011@ hotmail.com \\ Jeferson Gomes Moriel Junior \\ ORCID: https://orcid.org/ 0000-0003-1526-8002 \\ Instituto Federal de Mato Grosso, Brasil \\ E-mail: jeferson.moriel@cba.ifmt.edu.br
}

\begin{abstract}
Resumo
Na perspectiva de pesquisa sobre a base de conhecimento de professores, a disciplina da Biologia foi a pioneira entre as ciências a desenvolver um modelo sobre conhecimento especializado de professores transposto do modelo do Conhecimento Especializado de Professores de Matemática, o modelo do Conhecimento Especializado de Professores de Biologia (BTSK). Contudo, nosso objetivo foi identificar as conexões do conhecimento especializado de professores de Biologia e promover uma visão integrada das relações entre os subdomínios apresentados no modelo e que, para alcançarmos o nosso objetivo usamos como instrumento de análise PaP-eR (Professional and Pedagogical experience Repertoire) que relatassem práticas de ensino com conteúdo de citologia, sistema respiratório e interações ecológicas, configurando uma pesquisa de cunho qualitativo em que nossos resultados nos permitiu descrever uma visão integradora das conexões dos conhecimentos e compreender como todos os conhecimentos se articularam para fomentar os conhecimentos mais expressivo e fundamentador mobilizado no relato das práticas do Ensino Médio.
\end{abstract}

Palavras-chave: Conhecimento especializado de professores; Ensino de biologia; Conexões; BTSK.

\begin{abstract}
From the perspective of researching the knowledge base of teachers, the discipline of Biology was the pioneer among the sciences to develop a model of specialized knowledge of teachers transposed from the model of Specialized Knowledge of Mathematics Teachers, the model of Specialized Knowledge of Mathematics Teachers. Biology (BTSK). However, our objective was to identify the connections of the specialized knowledge of Biology professors and to promote an integrated view of the relationships between the subdomains presented in the model and that, in order to reach our objective, we used PaP-eR (Professional and Pedagogical Experience Repertoire) as an instrument of analysis. ) that reported teaching practices with content of cytology, respiratory system and ecological interactions, configuring a qualitative research in which our results allowed us to describe an integrative view of knowledge connections and understand how all knowledge articulated to promote more knowledge. expressive and grounding mobilized in the report of high school practices.
\end{abstract}

Keywords: Specialized knowledge of teachers; Biology teaching; Connections; BTSK.

\section{Resumen}

Desde la perspectiva de la investigación sobre la base de conocimientos de los docentes, la disciplina de Biología fue pionera entre las ciencias en desarrollar un modelo de conocimiento especializado de los docentes transpuesto del modelo de Conocimiento Especializado de los Docentes de Matemáticas, el modelo de Conocimiento Especializado de los Docentes de Matemáticas Biología (BTSK). Sin embargo, nuestro objetivo fue identificar las conexiones del conocimiento especializado de los profesores de Biología y promover una visión integrada de las relaciones entre los 
subdominios presentados en el modelo y que, para alcanzar nuestro objetivo, utilizamos PaP-eR (Professional y Repertorio de Experiencias Pedagógicas) como instrumento de análisis.) Que reportó prácticas docentes con contenido de citología, sistema respiratorio e interacciones ecológicas, configurando una investigación cualitativa en la que nuestros resultados nos permitieron describir una visión integradora de las conexiones del conocimiento y comprender cómo se articulaban todos los conocimientos. para promover conocimientos más expresivos y fundamentados movilizados en el informe de prácticas de secundaria.

Palabras clave: Conocimiento experto de professores; Enseñanza de biología; Conexiones, BTSK.

\section{Introdução}

Este trabalho está inserido em um movimento internacional iniciado nos anos 1980 sobre o knowledge base de professores para ensinar (Shulman, 1986) e que vem caminhando no sentido do desenvolvimento de tipologias e modelos que saem do genérico e focalizam o conjunto de conhecimentos especializados necessários ao professor de determinada matéria ou área do conhecimento (Moriel Junior \& Wielewski, 2017). Este é o caso dos estudos desenvolvidos pelo grupo SIDM ${ }^{1}$ na Universidad de Huelva, Espanha, que coordena a Red Iberoamericana MTSK. Tais estudos resultaram na criação do modelo Conhecimento Especializado de Professores de Matemática - MTSK ${ }^{2}$ que consiste em um marco teórico analítico-descritivo considerando as especificidades do ensino e da aprendizagem da matéria e suas diferentes naturezas, tanto do domínio matemático quanto no domínio didático do conteúdo matemático (Carrillo; Escudero \& Flores, 2014).

Nessa linha de pesquisa sobre a base de conhecimento de professores, a disciplina da Biologia foi a pioneira entre as ciências a transpor o modelo do MTSK, desenvolvendo o modelo Conhecimento Especializado de Professores de Biologia BTSK $^{3}$ (Luís, 2015), seguido dos modelos do Conhecimento Especializado de Professores de Física - PTSK ${ }^{4}$ (Lima, 2018) e do Conhecimento Especializado de Professores de Química - CTSK ${ }^{5}$ (Soares, 2019), e tendo como uma perspectiva atual a transposição para a Língua Portuguesa (Moreira, et al., 2020).

As investigações com a aplicação destes modelos como ferramentas metodológicas estão alcançando diversas áreas destas ciências e na Biologia as investigações estão voltadas em aprofundar na compreensão sobre quais conhecimentos especializados os professores têm ou devem ter para ensinar temas do ensino médio e de como esses conhecimentos são mobilizados durante a aula.

Os dados aqui apresentados fazem parte da dissertação da primeira autora, em que neste recorte enfatizamos as conexões de conhecimentos que consistem na relação de ligação entre os subdomínios que abrangem conhecimentos especializados manifestos em um episódio de ensino, seja ele em uma manifestação de um trecho bibliográfico, documental, ou advindo da transcrição de gravações audiovisuais. Essas conexões entre os conhecimentos especializados são amparadas na reflexão e construção de uma visão integradora de conceitos e propriedades a partir do raciocínio em busca de promover a formação, desenvolvimento e mobilização dos conhecimentos que permeiam os subdomínios dos conhecimentos pedagógicos e do conteúdo, caracterizando assim, o conhecimento especializado de professores de Biologia.

Para tanto, temos como objetivo identificar as conexões do conhecimento especializado de professores de Biologia e promover uma visão integrada das relações entre os subdomínios que, para alcançarmos o nosso objetivo usamos como instrumento de análise PaP-eRs (Professional and Pedagogical experience Repertoire) que relatassem práticas de ensino com conteúdo de citologia, sistema respiratório e interações ecológicas.

\footnotetext{
${ }^{1}$ Sigla em espanhol para: Seminario de Investigación en Didáctica de la Matemática liderado pelo Prof. Dr. José Carrillo Yáñez, Universidad Huelva, Espanha.

${ }^{2}$ Sigla em inglês para: Mathematics Teacher's Specialized Knowledge. Todas as siglas deste modelo foram definidas em inglês por seus autores.

${ }^{3}$ Sigla em inglês padronizada pela Red Iberoamericana para: Biology Teacher's Specialized Knowledge.

${ }^{4}$ Sigla em inglês padronizada pela Red Iberoamericana para: Physical Teacher's Specialized Knowledge.

${ }^{5}$ Sigla em inglês padronizada pela Red Iberoamericana para: Chemistry Teacher's Specialized Knowledge.
} 


\section{Conhecimento Especializado de Professores de Biologia}

O modelo do Conhecimento Especializado de Professores de Biologia (BTSK) (Luís, 2015) (Figura 1), segue o formato hexagonal em consonância com o modelo do Conhecimento Especializado de Professores de Matemática - MTSK (Carrillo et al., 2013).

Por ser considerado o modelo da Biologia em estado de construção por Luís (2015), seu atual cenário em desenvolvimento não apresenta a descrição de todas as categorias dos subdomínios, nem a descrição das crenças dos professores sobre o Conhecimento da Biologia e do Conhecimento Pedagógico do Conteúdo, que no MTSK se encontra no centro do modelo, nem pouco traz à luz fundamentos peculiares do ensino de Biologia, sendo este modelo considerado atualmente como uma transposição direta do MTSK. Para tanto, a fundamentação teórica para os indicadores das categorias, emergiram de revisões da literatura e dos dados analisados.

Figura 1 - Modelo do Conhecimento Especializado dos Professores de Biologia - BTSK.

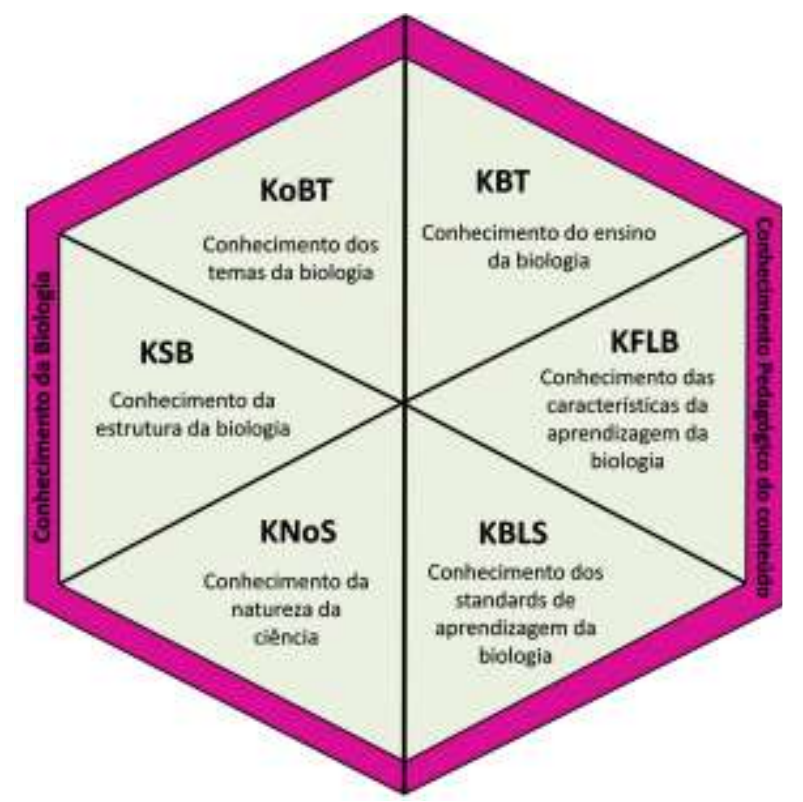

Fonte: Luís (2015).

Apresentamos a seguir, os três subdomínios pertencentes ao domínio do Conhecimentos da Biologia (BK). Embora todos os subdomínios tenham suas definições estabelecidas, é importante destacar que atualmente, apenas um desses subdomínios (o Conhecimento dos Temas da Biologia - KoBT) possui descritas explicitamente suas categorias internas.

O subdomínio Conhecimento dos Temas da Biologia (KoBT) diz respeito ao conhecimento das definições, fenômenos, teorias, leis, representações e aplicações dos conteúdos da Biologia (Luís, 2015).

Atualmente existem seis categorias pertencentes a esse subdomínio (Luís, 2015), a saber:

1. Conhecimento de Conceitos e de Exemplos Associados - Conhecimento das definições ou propriedades específicas que caracterizam os elementos ou conceitos biológicos e dos exemplos que ajudam a defini-lo.

2. Conhecimento de Leis, Princípios e Teorias - Conhecimento das leis, princípios e teorias associadas aos conteúdos de Biologia.

3. Conhecimento dos Fatos e Fenômenos - Conhecimento sobre fatos e fenômenos como verdades dogmáticas e dos fenômenos biológicos enquanto processos e sequências de acontecimentos biológicos.

4. Conhecimento de Procedimentos e Técnicas de Observação - Conhecimento sobre os meios e técnicas apropriadas para realizar determinada observação mas também sobre como e quando fazer. 
5. Conhecimento de Modelos Relacionados com o Conteúdo - Conhecimento sobre estruturas ou registros que permitem diferentes representações de um determinado conteúdo.

6. Conhecimento sobre Aplicações do Conteúdo - Conhecimento de uma ampla variedade de contextos e aplicações ligada ao conteúdo, que permitem ao professor conhecer os diferentes significados que se podem atribuir ao conteúdo.

O subdomínio Conhecimento da Estrutura da Biologia (KSB) é o conhecimento da estrutura da Biologia, das relações entre os diferentes conteúdos (Luís, 2015).

O subdomínio Conhecimento da Natureza da Ciência (KNoS) relata sobre o conhecimento da natureza, dos princípios e das grandes ideias sobre ciências, de ciência e em ciências, como também aborda acerca dos métodos e procedimentos científicos, podendo ser manifesto pelo professor durante a realização de uma aula prática.

Detalharemos a seguir os três subdomínios do domínio do Conhecimento Pedagógico do Conteúdo (PCK) do BTSK.

O subdomínio Conhecimento do Ensino de Biologia (KBT) diz respeito ao conhecimento de estratégias de ensino da Biologia, como o uso dos recursos didático e material, a microscopia, trabalho experimental, aulas de campo, exposição dialógica, questionários, uso e/ou montagem de modelos tridimensionais e maquetes, e uso de analogias, e fragilidades e fortalezas do ensino.

O subdomínio Conhecimento das Características da Aprendizagem da Biologia (KFLB) que é o conhecimento da forma como os alunos aprendem Biologia, o trabalho prático como elemento facilitador, fortalezas e fragilidades da aprendizagem, alternativas como desbloqueio às novas aprendizagens.

O subdomínio Conhecimento dos Standards de Aprendizagem da Biologia (KBLS) que é conhecimento dos parâmetros de aprendizagem da Biologia, normas e programas instrucionais, estrutura curricular, complementos ao currículo, estudos complementares, relações ontológicas.

Em suma, o BTSK apresenta as seguintes propriedades: no domínio do Conhecimento da Biologia (BK) o elemento fundamental é o conhecimento que o professor tem ou deve ter do conteúdo a ser abordada, e o domínio do Conhecimento Pedagógico do Conteúdo (PCK) é fundamentado nas ações, decisões e parâmetros exigidos pela profissão docente para o processo de ensino e aprendizagem.

\section{Encaminhamento Metodológico}

De natureza qualitativa (Bogdan \& Biklen, 1982) e bibliográfica cuja a fonte de dados é a produção científica classificada como Professional and Pedagogical experience Repertoire (PaP-eR) que par tal, recorremos ao repositório digital Google Acadêmico para seleção dos PaP-eR e especificamos quais critérios de inclusão determinariam os PaP-eRs que comporiam o objeto de estudo. Contudo, os critérios de inclusão foram: Idioma; Período; Palavras-chave.

Loughran et al. (2001) definiu PaP-eR como relato de experiência de ensino que apresenta conhecimento de professores mobilizados na prática e descrições detalhadas das situações vivenciadas durante o ensino de um determinado conteúdo que permite o reconhecimento de aspectos didáticos, do conteúdo e do contexto, abordando aspecto experimental do ensino da disciplina.

O primeiro passo para obtenção dos dados foi a identificação dos episódios de ensino, definidos por Loughran et. al. (2001) como informações significativas ao leitor/pesquisador que são validadas e autenticadas pelo professor e reconstruído por escrito ou obtidas em sala de aula. Para tanto, os conhecimentos identificados foram codificados com uma sequência alfanumérica indicando o símbolo "§" e o número de identificação do Parágrafo, e a letra "L" seguida da numeração separada por traço para identificar a Linha, sendo que a numeração das linhas é sequencial, iniciando-se a cada parágrafo. Neste trabalho, analisamos um três PaP-eRs identificado como P1, P2 e P3 e a codificação seguirá o seguinte exemplo: P1.§9.L1-5. 
Após a identificação dos episódios e suas respectivas codificações, o passo seguinte foi caracterizar os conhecimentos especializados dos professores de Biologia usando o modelo analítico do BTSK (cf. Figura 1). Os mesmos foram desmembrados em "evidências" de conhecimentos, que é quando o episódio por si só comprova qual ou quais conhecimentos foram mobilizados pelo professor, e em "indícios" que são episódios que demonstram uma superficialidade de um determinado conhecimento sugerindo que o sujeito possa saber mais sobre o que foi exposto com o intuito de converter em evidência de conhecimento (Moriel Junior \& Carrillo, 2014; Flores-Mendrano, 2015).

Para a caracterização dos conhecimentos especializados e identificação das conexões, baseamos no instrumento de análise MTSK (Moriel Junior \& Alencar, 2019) seguindo um percurso pautado em: Conhecimento (subdomínio) > Associado a (categoria) > Que consiste em (síntese do conhecimento) (cf. Figura 2).

Figura 2 - Instrumento de análise MTSK.

\begin{tabular}{|cccc|}
\hline TRECHO DO ARTIGO & & \multicolumn{2}{c|}{ ANALISE DO PESQUISADOR } \\
\hline Evidência & Conhecimento... & associado a.... & que consiste em... \\
\hline $\begin{array}{c}\text { [Trecho do episódio - linha } \\
\text { ou página, artigo, ano] }\end{array}$ & [subdominio] & [categoria] & [Sintese do conhecimento] \\
\hline $\begin{array}{c}\text { Exemplo: "eu utilizo a } \\
\text { resolução de problemas para } \\
\text { ensinar derivada" } \\
\text { (Artigo, Ano, página) }\end{array}$ & $\begin{array}{c}\text { do ensino de } \\
\text { matemática (KMT) }\end{array}$ & $\begin{array}{c}\text { estratégias de } \\
\text { ensino }\end{array}$ & $\begin{array}{c}\text { uma abordagem de } \\
\text { resolução de problemas } \\
\text { para ensinar derivadas }\end{array}$ \\
\hline
\end{tabular}

Fonte: Moriel Junior e Alencar (2019).

Portanto, as conexões não estão obrigatoriamente vinculadas à quantidade de vezes que foram identificadas, mas sim sobre sua relevância na mobilização dos conhecimentos especializados durante o processo ensino-aprendizagem.

Para ilustração dessa pesquisa, utilizaremos figuras geométricas hexagonais onde identificaremos por setas as conexões formadas pelos respectivos conhecimentos especializados.

\section{Resultados e Discussão}

Trazemos à luz da discussão a visão integradora das conexões entre os subdomínios dos conhecimentos especializados na intenção de corroborar com as necessidades formativas e da prática profissional sobre a investigação de quais conhecimentos especializados mobilizados que os professores de Biologia têm ou devem ter para ensinar temas abordados no Ensino Médio. Portanto, tais conexões entre os subdomínios amparam que processo de ensino-aprendizagem acontece à medida que os conhecimentos especializados se relacionam entre si durante a mobilização do conteúdo em sala de aula permeando entre os Conhecimentos da Biologia (BK) e dos Conhecimentos Pedagógico do Conteúdo (PCK).

\section{PaP-eR 1. Práticas no Ensino de Biologia: Conhecendo a Célula do Tecido da Cebola}

Esse PaP-eR relata uma aula prática de Citologia com intuito de chamar atenção dos alunos e propiciar a eles uma aproximação com uso de recursos didáticos e métodos científicos oportunizar a identificação das estruturas celulares da raiz da cebola (Allium cepa). Para tanto, adotaram a metodologia do corte de mão livre que possibilitou aos alunos prepararem as lâminas histológicas utilizando corantes para visualizações no microscópio e assim abrir uma porta para o conhecimento do mundo científico. Posteriormente, construíram maquetes e explicaram sobre o que aprenderam no laboratório com a aula prática (Paiva, et al., 2018).

Dos trinta e oito conhecimentos especializados de professores de Biologia identificados nesse PaP-eR temos que, apesar da assimetria da quantidade de conhecimentos por subdomínio, o Conhecimento das Características da Aprendizagem 
da Biologia (KFLB) além de ter sido o conhecimento mais identificado, também foi bastante expressivo entre as conexões dos conhecimentos, que totalizaram nove.

O que sustenta o Conhecimento das Características da Aprendizagem da Biologia (KFLB) ser o conhecimento mais expressivo é o fato de que a estratégia de ensino adotada para abordar os conceitos e definições do tema sobre Citologia Vegetal estavam voltadas para facilitar a aprendizagem e oportunizar novos conhecimentos científicos aos alunos, como podemos ver na Figura 3.

Figura 3 - Conexões entre os Conhecimentos Especializados no PaP-eR 1.

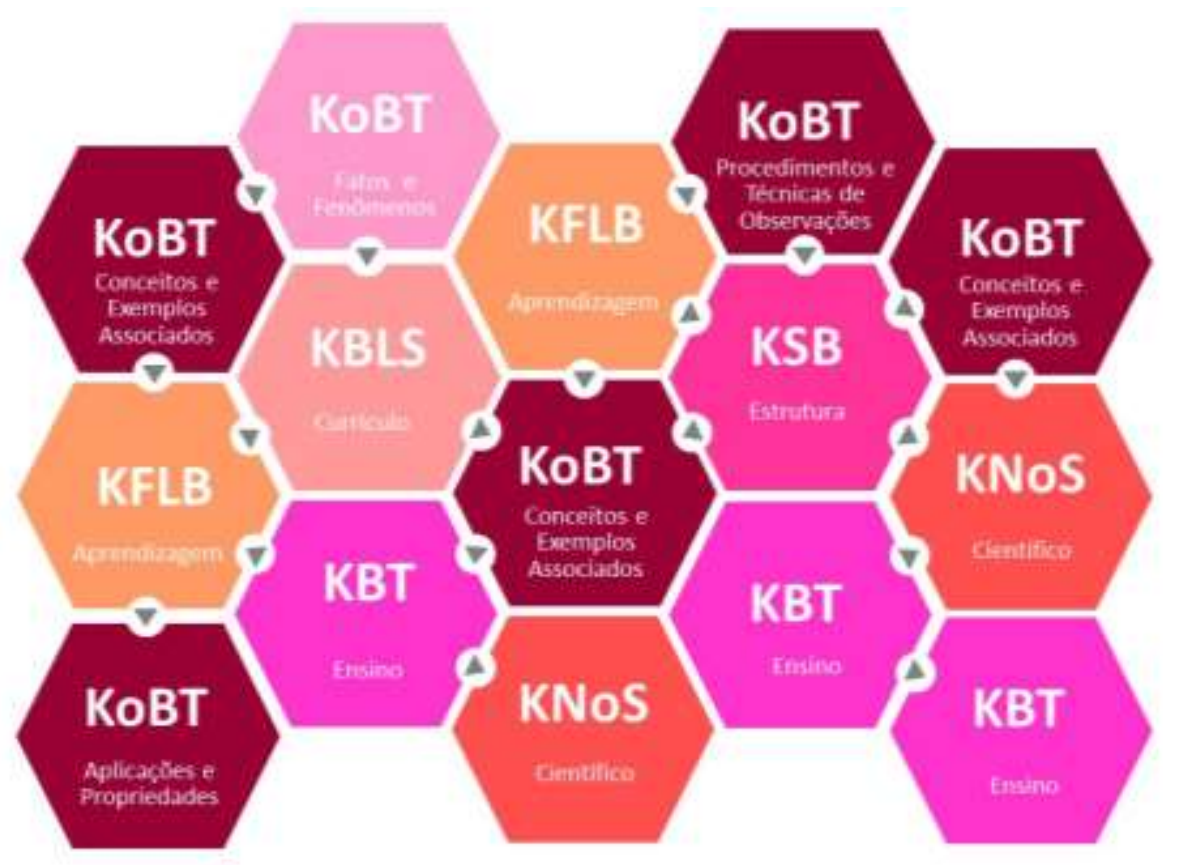

Fonte: Marques (2020).

A respeito da forma como os alunos aprendem (Conhecimento das Características da Aprendizagem da Biologia KFLB) o trecho P1.§23.L6-10 que consiste na característica do trabalho prático como alternativo e atrativo: 'os mesmos (alunos) diziam que estava maravilhados e não sabiam como é magnífico ver um mundo que pra eles outrora não passavam de meras ilustrações em livros ou vídeos.', é fundamentado na literatura por Orlando et al. (2009) ao dizerem que o envolvimento dos alunos nas atividades que utilizam metodologias diversas como modelos didáticos e ilustrações proporciona aos alunos uma melhor assimilação dos conteúdos e com isso promove o conhecimento aumentando o rendimento de forma significativa.

\section{PaP-eR 2. Relato de Experiência: Trabalhando Projetos de Ensino em Estágio Supervisionado}

O referido PaP-eR traz a importância do Estágio Supervisionado na formação docente unindo a teoria aprendida durante o curso com a prática na formação por meio de um Projetos de Ensino. Entretanto, o Projeto de Ensino intitulado "Sistema Respiratório: anatomia, fisiologia e a influência do meio ambiente externo" possuiu como público-alvo, alunos do ensino médio e foi baseado em dois livros didáticos e, devido à flexibilidade do planejamento, o projeto sofreu alterações durante a sua execução, de forma que os conteúdos e atividades fossem reorganizadas possibilitando trabalhar utilizando infográficos, atividade experimental e simulação. A interação entre os alunos foi perceptível e vinculada ao uso de metodologias diferenciadas (Santos, 2016).

O PaP-eR e sobre Sistema Respiratório apresentou uma maior mobilização do Conhecimento da Estratégia de Ensino 
(KBT) quando o professor utilizou o Projeto de Ensino como uma estratégia oportuna para desenvolver, junto com os pibidianos, aulas práticas para avaliação do ar e com confecção de material que também seria manipulado durante as aulas expositivas com intuito de facilitar a aprendizagem e envolver os alunos com os temas interdisciplinares.

Notamos que os demais conhecimentos, principalmente os mais presentes entre as conexões (Conhecimentos das Características da Aprendizagem da Biologia - KFLB, Conhecimentos dos Temas da Biologia - KoBT) sustentaram a mobilização do Conhecimento do Ensino da Biologia (KBT) como explicito no trecho P2.§18.L1-4: 'Foi notável que a participação dos alunos tornou-se mais efetiva quando a aula não estava "presa" aos slides. Observou-se também que a aula com os slides se tornava mais rápida, menos interativa e muitas vezes se limitava ao que estava exposto na figura. Com o quadro, era possível retornar a outras partes, desenhar, apagar e redesenhar estruturas que fossem importantes.' Assim como traz a evidência do trecho P2.\$21.L1-5 que consiste características da atividade de experimentação na forma dos alunos discutirem, questionarem e analisarem as possíveis soluções para o problema, e o trecho: "Foi executado a parte inicial do experimento "avaliando a qualidade do ar". Onde foram colocados 2 materiais em locais aleatórias da escola, escolhido pelos próprios discentes. Este tipo de atividade se caracteriza como um importante instrumento alternativo que se distanciam do ensino tradicional. Tende, também a melhorar a aprendizagem e intensifica o papel do discente na atividade" (P2.§15.L5-8).

Figura 4 - Conexões entre os Conhecimentos Especializados no PaP-eR 2.

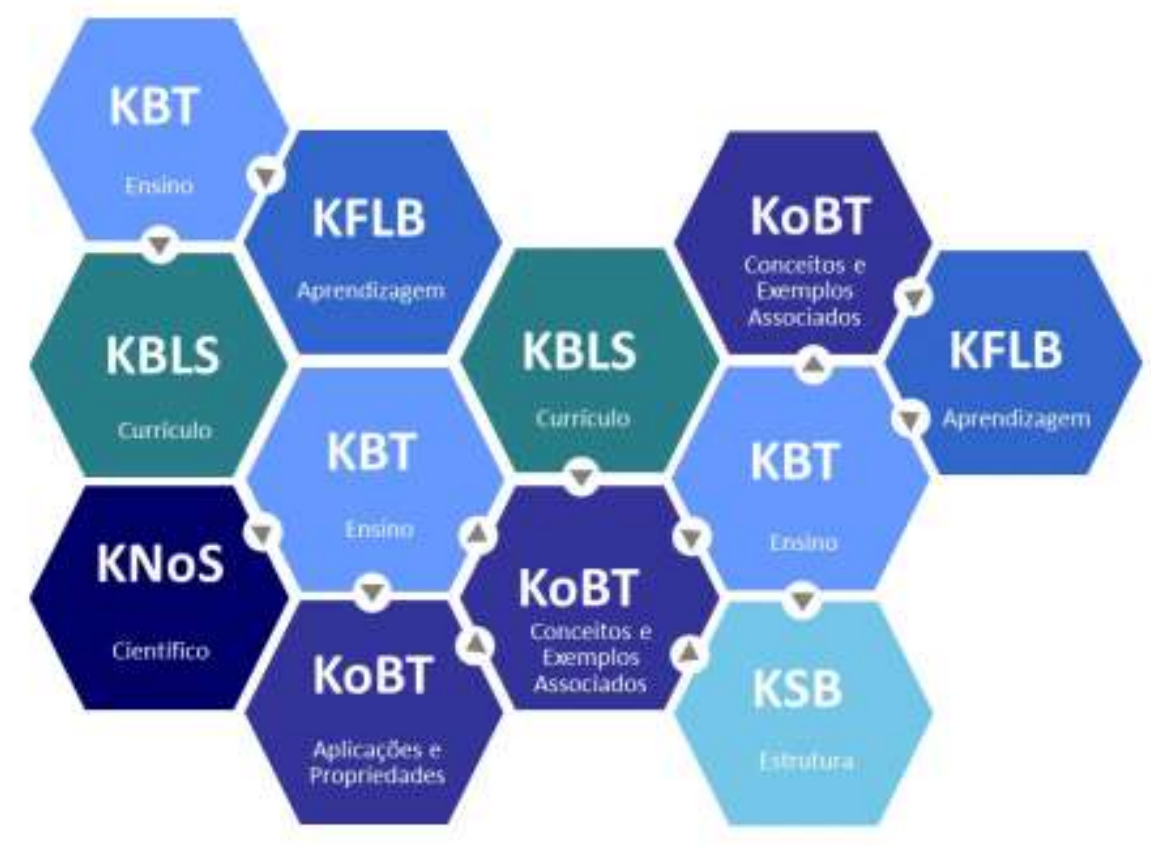

Fonte: Marques (2020).

\section{PaP-eR 3. Abordagem do Conteúdo Interações Ecológicas no Contexto do PIBID: Utilizando uma Atividade Prática como}

\section{Ferramenta Facilitadora no Processo de Ensino e Aprendizagem}

Este PaP-eR relata uma atividade prática intitulada "Interações Ecológicas" desenvolvida com o objetivo de propiciar a fixação do conteúdo de Interações Ecológicas por parte dos alunos de três turmas. A dinâmica da atividade se deu da seguinte forma: os alunos se dividiram em seis grupos, e cada grupo ficou sob a supervisão de um bolsista do Programa Institucional de Bolsa de Iniciação à Docência (PIBID). Cada bolsista explanou sobre um tipo de bioma brasileiro para os seus respectivos grupos e após a explanação os grupos receberam plaquinhas com nomes de animais de cada bioma, sinais de positivo e negativo, nomes de tipos de interações ecológicas e setas feitas com cartolina e barbante. Com o material em mãos, os alunos deveriam montar uma teia alimentar onde as setas deveriam apontar para o animal que seria a presa e os sinais iriam dizer se 
aquela relação era harmônica ou desarmônica. Ao final da montagem da teia, cada grupo deveria socializar a sua teia com os colegas. (Damasceno et al., 2017).

Dos trinta e sete conhecimentos especializados de professores de Biologia caracterizados nesse PaP-eR, três subdomínios se destacam caracterizando dez conexões entre eles: Conhecimentos do Temas da Biologia (KoBT), do Ensino da Biologia (KBT) e o das Características da Aprendizagem da Biologia (KFLB).

Partindo da premissa que os conhecimentos do Temas da Biologia (KoBT), do Ensino da Biologia (KBT) e o das Características da Aprendizagem da Biologia (KFLB) foram os conhecimentos mais mobilizados durante a aula relatada nesse PaP-eR sobre Interações Ecológicas, percebemos que os mesmos vão ao encontro do objetivo da aula: o uso de estratégias de ensino voltadas para uma aula prática baseada no lúdico como atividade potencializadora contribuísse para aprendizagem durante a exploração de um tema específico "Interações Ecológicas". O trecho P3.§26.L1-3 vigora essa conexão entre os subdomínios dos conhecimentos quando diz que: 'Ao final da montagem, cada grupo foi orientado a socializar a sua teia com os outros grupos, mencionando o bioma ao qual ela pertencia, suas características principais, o nível trófico do qual cada animal fazia parte e o tipo de relação existente entre eles' que consiste na interação entre os alunos como forma alternativa de aprendizado: 'cada grupo foi orientado a socializar' (KFLB), nos conceitos dos biomas e definições das principais características e o nível trófico de cada animal: 'mencionando o bioma ao qual ela pertencia, suas características principais, o nível trófico do qual cada animal fazia parte e o tipo de relação existente entre eles' (KoBT) e na última etapa da atividade prática sobre IE: socialização dos resultados (teias) com outros grupos (KBT).

As conexões identificadas nesse PaP-eR (Figura 5) nos permite sustentar a ideia de o conhecimento mais mobilizado (Conhecimento dos Temas da Biologia - KoBT) é o conhecimento que amparou a estratégia de ensino mobilizada pelo professor (Conhecimento do Ensino de Biologia - KBT) pois a condição de conhecer o conteúdo, a dinâmica existente em seus tópicos, a complexidade dos detalhes que distinguem uma relação ecológica da outra, as características dos biomas e das propriedades dos níveis tróficos, e como todas essas peculiaridades se relacionam no ecossistema sustentando comunidades e populações de seres vivos, considerando que os conceitos e definições se estabelecem pela interação intraespecífica e interespecífica conduziu o professor a sensibilizar-se às características implícitas às estratégias de ensino, como aula prática, utilizando do lúdico como um recurso didático e motivador para alcançar o objetivo principal de uma aula: a aprendizagem.

Figura 5 - Conexões entre os Conhecimentos Especializados no PaP-eR 3

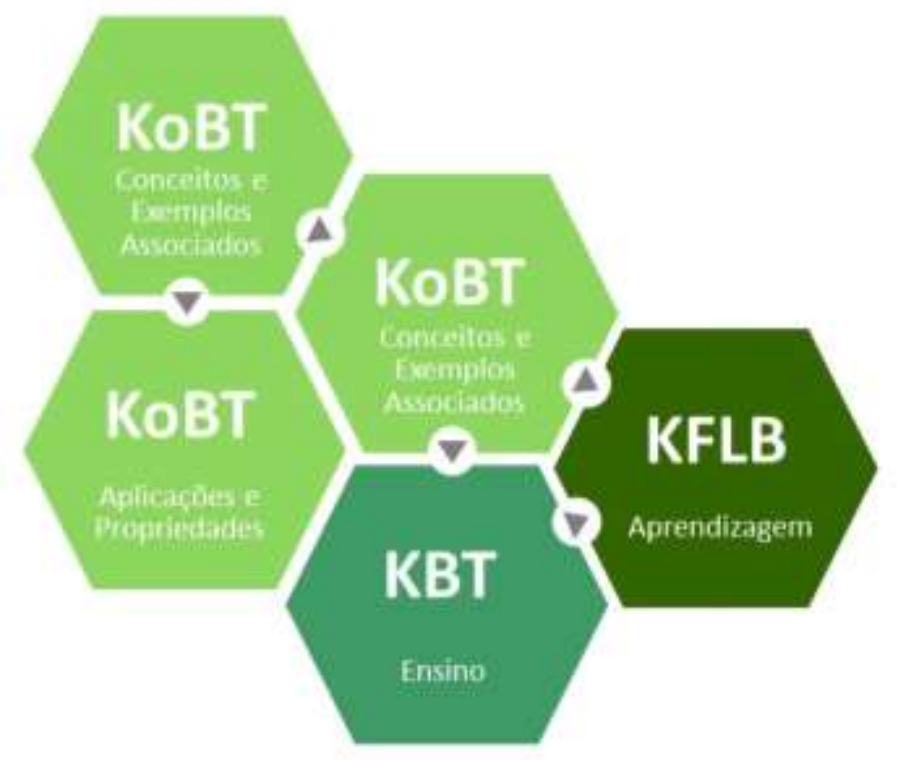

Fonte: Marques (2020). 
Ao compreender o conteúdo de Interações Ecológicas (KoBT) os alunos apreendem não só conceitos biológicos, mas também sobre sua própria relação (KBLS) como um ser natural com outros seres bióticos. Esse trecho nos permite dizer sobre um nível de desenvolvimento conceitual e ontológico (a ser) desenvolvido nessa etapa escolar sobre interações ecológicas: 'conceitos biológicos' e noções 'sobre sua própria relação como um ser natural com outros seres bióticos.':

Também torna possível o reconhecimento do aluno como organismo e parte integrante da Natureza e, portanto, sujeito aos mesmos processos, fenômenos e interações que os demais seres vivos, e capaz de modificar ativamente a biodiversidade e as relações estabelecidas entre os organismos (Brasil, 2006 como citado em Quesado, 2009).

Buscando resposta à pergunta norteadora dessa investigação, a análise dos dados de conhecimentos especializados de professor de Biologia sobre o conteúdo de Interações Ecológicas, nos permite dizer que os Conhecimentos do Temas da Biologia (KoBT), do Ensino da Biologia (KBT) e o das Características da Aprendizagem da Biologia (KFLB) mobilizados nesse PaP-eR fundamentam a prática docente sobre este conteúdo quando ministrado no Ensino Médio.

\section{Visão Integradora dos Três PaP-eRs - Conexões de Conhecimentos}

Durante a prática de ensino, o professor mobiliza diversos saberes (Tardif 2002, 2008), habilidades (Gauthier, 1998) e conhecimentos (Shulman, 1986, 1987), sejam esses generalistas ou especialistas, cabendo ao professor analisar o conteúdo considerando a vivência dos alunos, assumindo um papel reflexivo à sua prática metodológica, às formas de aprendizagem de seus alunos e o quanto eles estarão envolvidos com o conteúdo. Portanto, cabe ao professor assumir o papel de pesquisador ao refletir sobre sua prática e como a mesma contribuirá com o produto final do ensino: a aprendizagem (Cavalcanti \& Moita Lopes, 1992).

A visão integrada dos três PaP-eRs analisados (cf. seções anteriores) nos permitiu identificar quais conhecimentos se conectaram mais expressivamente construindo as práticas relatadas sobre abordagens dos conteúdos do Ensino Médio visto que, por vezes, a caracterização de um determinado conhecimento, como por exemplo, o Conhecimento dos Standards de Aprendizagem da Biologia (KBLS), atuando como propulsor da mobilização dos demais conhecimentos que, por meio das conexões, deram o sentido à aula.

Avançando em nossos resultados, integramos as conexões dos conhecimentos mais expressivos que sustentaram as práticas mobilizadas e relatadas nos três PaP-eRs (Figura 6) com intuito de sintetizar as conexões entre os conhecimentos e, com isso, notamos que, mesmo que a dispersão dos conhecimentos tenham acontecido de forma assimétrica e o grau de expressividade distinto sendo também mobilizados outros conhecimentos, as conexões dos conhecimentos nos três PaP-eRs ocorreram expressivamente entre os conhecimentos do Ensino da Biologia (KBT), das Características da Aprendizagem da Biologia (KFLB) e dos Temas da Biologia (KoBT), sendo neste último, mobilizados quatro das seis categorias: Conceitos Exemplos Associados, Fatos e Fenômenos, Procedimentos e Técnicas de Observação e Aplicações do Conteúdo.

Corroborando aos nossos resultados e às perspectivas dos domínios do conhecimento pedagógico e do conteúdo do modelo BTSK, Bolzan (2009) caracteriza o conhecimento pedagógico como saber os conceitos, esquemas práticos de ensino, estratégias pedagógicas, experiências do professor cultural, institucional e escolar, e o conhecimento disciplinar ou da matéria ensinada o conhecimento da formação acadêmica e da prática de ensino. 


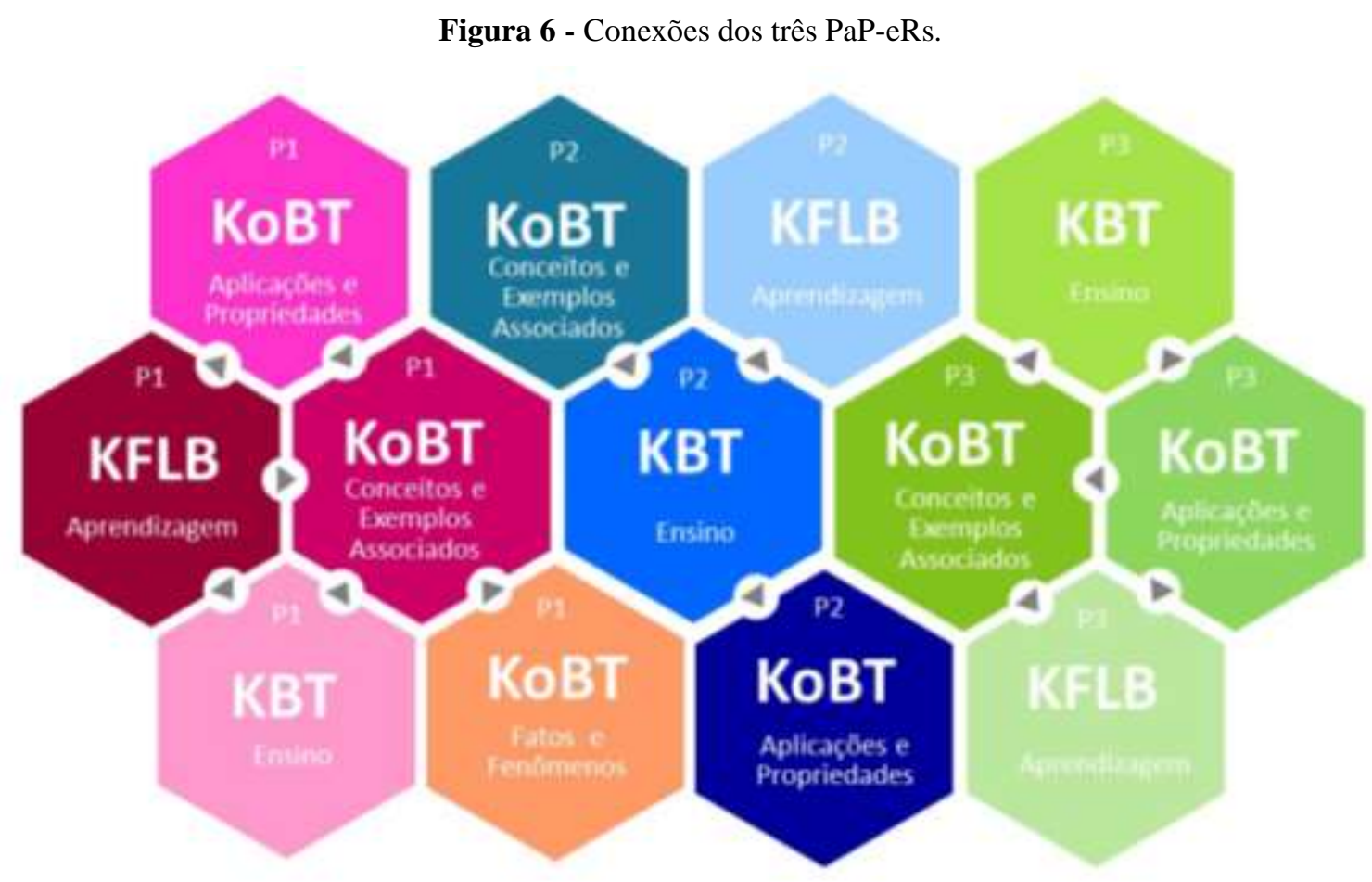

Fonte: Marques (2020). Adaptado pelos autores.

Todavia, o que configura a especialização do conhecimento não diz respeito apenas a quais conhecimentos estão sendo mobilizados em um determinado cenário, mas também em como esses conhecimentos se tornam especializados do professor de Biologia ao estabelecerem as conexões entre eles e ao permearem durante a prática docente os conhecimentos especializados dos domínios do Conhecimento da Biologia (BK) e do Conhecimento Pedagógico do Conteúdo (PCK).

\section{Considerações Finais}

Apesar dos nossos resultados da análise estarem restritos ao atual estado de conhecimento sobre o modelo BTSK, alcançamos, como proposto nessa pesquisa, a caracterização dos conhecimentos especializados dos professores de Biologia mobilizados nos três PaP-eRs. O avanço da pesquisa se deu com a identificação e discussão das conexões entre os subdomínios dos conhecimentos especializados por meio de uma visão integradora acerca dos conhecimentos que sustentaram as práticas relatadas sobre os conteúdos de Biologia. Essa visão integradora das conexões dos conhecimentos nos permite compreender como todos os conhecimentos se articularam para fomentar os conhecimentos mais expressivo e fundamentador mobilizado no relato das práticas do Ensino Médio.

Considerando nossos achados, parece razoável que possa interessar aos docentes em exercício como uma atividade de autorreflexão de sua prática, de seus saberes e habilidades, aos licenciandos de Ciências Biológicas e Ciências da Natureza (habilitação em Biologia) como ferramenta inovadora para uso em suas futuras práticas. Contudo, os resultados também podem ter aplicações práticas nos grupos de estudos sobre o conhecimento especializado e grupos formadores de professores de Biologia.

Uma das limitações deste trabalho é o atual cenário do modelo, principalmente no que diz respeito às descrições das categorias dos demais subdomínios, pois só temos as descrições das categorias do Conhecimento dos Temas da Biologia (KoBT).

Outros trabalhos são necessários para corroborarem nossos resultados quanto ao uso do modelo como uma ferramenta analítica e metodológica que, para tal, sugerimos o uso de métodos empíricos (gravação audiovisual de aulas de Biologia) 
assim como, estender as pesquisas com os sujeitos sendo licenciandos e professores que atuam no Ensino Superior.

\section{Agradecimentos}

Os autores agradecem e dedicam ao Prof. Dr. José Carrillo (querido Pepe) (in memoriam) pela dedicação, incentivo, acolhimento, amizade e ensinamentos.

Os autores agradecem também ao apoio da Coordenação de Aperfeiçoamento de Pessoal de Nível Superior - Brasil (CAPES) - Código de Financiamento 001, da FAPEMAT (Edital Universal 42/2016/FAPEMAT) e do IFMT (Chamadas 04/2018 e 01/2019/DPG/PROPES/IFMT).

\section{Referências}

Begon, M., Colin R., Townsende, J. \& Hasper L (2007). Tradução Adriano Sanches Ecologia de Indivíduos a Ecossistemas. (4a ed.) Artmed Editora.

Bogdan, R. C. \& Biklen, S. K. (1982). Qualitative research for education, an introduction for to theory and methods. Boston, Allyn and Bacon, 27-30.

Bolzan, D. P. V. Formando professores reflexivos. (2009). In.: Formação de Professores: Compartilhando e Reconstruindo Conhecimentos (2a ed.) Mediação.

Carrillo, J., Contreras, L. C. \& Flores, P. (2013). Un modelo de conocimiento especializado del profesor de matemáticas. Rico, L. C., Añadas, M., Gutiérrez, J., Molina, M., Segovia, I., (Eds.), Investigacion en Didáctica de la Matemática. Homenaje a Encarnación Castro. Granada: Editorial Comares, 193-200.

Carrillo, J., Avila, D. I. E., Mora, D. V. \& Medrano, E. F. (2014). Un marco teórico para el conocimiento especializado del professor de matemáticas, Universidad de Huelva Publicaciones.

Carrillo, J., Escudero, D. I. \& Flores, E. (2014). El uso del MTSK em la formación inicial de profesores de matemáticas de primaria. For-Mate 1(16).

Cavalcanti, M. \& Moita Lopes, L. P. (1992). Implementação de Pesquisa na Sala de Línguas no Contexto Brasileiro. In.: Trabalhos de Linguística Aplicada. Campinas.

Damasceno, I. T., Guimarães, M. S., Pereira, N. A. \& Almeida, O. da S. (2017). Abordagem do Conteúdo Interações Ecológicas no Contexto do PIBID: utilizando uma atividade prática como ferramenta facilitadora no processo de ensino e aprendizagem. In: Seminário Gepráxis Vi Seminário Nacional II Seminário Internacional Políticas Públicas, Gestão e Práxis Educacional, 2017, Vitótia da Conquista, BA. Anais... Seminário GEPRÁXIS, Vitótia da Conquista, BA 6 (6), 1661-1672. http://anais.uesb.br/index.php/semgepraxis/issue/current.

Gauthier, C. (1998). Por uma teoria da Pedagogia: pesquisas contemporâneas sobre o saber docente. Ed. Unijuí,Ijuí, RS.

Lima, S. S. (2018). Conhecimento Especializado de Professores de Física: Uma proposta de Modelo Teórico. 144 f. Dissertação (Mestrado em Ensino). Programa de Pós-graduação em Ensino, Instituto Federal de Educação, Ciências e Tecnologias do Estado de Mato Grosso, Cuiabá.

Luís, M. (2015). Conhecimento Especializado de Professores de Biologia. Tese (Doutorado). Univesidad de Huelva, Espanha (Documento Inédito).

Loughran, J., Milroy, P., Berry, A., Gunstone, R. \& Mulhall, P. (2001). Documenting science teachers' pedagogical content knowledge through PaP-eRs. Research in Science Education, 31 (2), 289-307.

Marques, M. (2020). Conhecimento Especializado de Professores de Biologia: análise de relatos de prática no Ensino Médio. 110 f. Dissertação (Mestrado em Ensino). Programa de Pós-graduação em Ensino, Instituto Federal de Educação, Ciências e Tecnologias do Estado de Mato Grosso, Cuiabá.

Moreia, J. S. S.; Silva, M. M. \& Evangelista, E. G. (2020). Conhecimento especializado de professores de Língua Portuguesa PLTSK1: transposição direta do MTSK. Research, Society and Development, 9, (11), e749119513, http://dx.doi.org/10.33448/rsd-v9i11.9513. https://rsdjournal.org/index.php/rsd /article/view/9513

Moriel Junior, J.G. \& Carrillo, J. (2014). Explorando indícios de conhecimento especializado para ensinar matemática com o modelo MTSK. In: Seminário De Investigación en Educación Matemática, 18., 2014, Salamanca, Espanha. Anais... Salamanca, Espanha, 1-10.

Moriel Junior, J. G. \& Wielewski, G. D. (2017). Base de conhecimento de professores de matemática: do genérico ao especializado. Revista de Ensino, Educação e Ciências Humanas, 18 (2), 126-133.

Moriel Junior, J. G., \& Alencar, A. P. (2019). Conhecimento especializado para ensinar Cálculo: um panorama da produção do COBENGE $2012-2017$. Brazilian Journal of Development, 5(7), 7687-7702.

Orlando, T.C.; Lima, A.R.; Da Silva, A.M.; Fuzissaki, C.N.; Ramos, C.L.; Machado, D.; Fernandes, F.F.; Lorenzi, J.C.C.; Lima. M.A.; Gardim, S.; Barbosa, V.C. \& Tréz, T.A. (2009). Planejamento, montagem e aplicação de modelos didáticos para abordagem de biologia celular e molecular no ensino médio por graduandos de ciências biológicas. Revista Brasileira de Ensino de Bioquímica e Biologia Molecular, 1 (1), 1-17.

Paiva, F. G. B.; Silva, A. M.; Paiva, T. I. F. \& Almeida, L. M. (2018). Práticas no Ensino de Biologia: Conhecendo a Célula do Tecido da Cebola. In: Congresso Nacional De Pesquisa E Ensino Em Ciêcnias, III., 2018, Campina Grande, PB. Anais... III CONAPESC, Campina Grande, PB, 1.

Quesado, L. B. (2009). Interações Ecológicas nos Livros Didáticos do Ensino Médio. - Rio de Janeiro, 2009. Instituto de Biologia Roberto Alcântara Gomes da Universidade do Estado do Rio de Janeiro, como requisito para obtenção de grau de licenciada em Ciências Biológicas, pp. 1-114. 
Research, Society and Development, v. 10, n. 12, e269101220424, 2021

(CC BY 4.0) | ISSN 2525-3409 | DOI: http://dx.doi.org/10.33448/rsd-v10i12.20424

Santos, Y. S. (2016). Relato de Experiência: trabalhando projetos de ensino em estágio supervisionado. Revista de Pesquisa Interdisciplinar. ISSN 2526-3560, 1, pp. 437-443.

Shulman, L. (1986). Those who understand: Knowledge growth in teaching. Educational researcher, 15 (2), pp. 4-14,

Shulman, L. (1987). Knowledge and teaching: Foundations of the new reform. Harvard educational review, 57 (1), 1-23.

Soares, S. T. C. (2019). Conhecimento Especializado de Professores de Química - CTSK: Proposta de Modelo Teórico. 88 f. Dissertação (Mestrado em Ensino). Programa de Pós-Graduação Stricto Sensu em Ensino, Instituto Federal de Educação, Ciência e Tecnologia de Mato Grosso, Cuiabá.

Tardif, M. (2002). Saberes docentes e formação profissional. (4a ed.) Vozes.

Tardif, M. (2008). Saberes docentes e formação profissional. (9a ed.) Vozes. 